\title{
EVALUACIÓN IN VITRO DE LA ACTIVIDAD ANTIHEMOLÍTICA DE EXTRACTOS ACUOSOS (CAMELLIA SINENSIS, HIBISCUS SABDARIFFA LINN), BAJO UN DISEÑO FACTORIAL 2X5
}

\section{In vitro evaluation of the anti-haemolytic activity of aqueous extracts (Camellia sinensis, Hibiscus sabdariffa Linn), under a 2x5 factorial design}

\author{
Franklin Pacheco Coelloa, Ibis Pinto-Catari ${ }^{b}$, Doralys Ramirez-Azuajec, \\ Corymar Orosco-Vargas ${ }^{d}$ y María Peraza-Marrero
}

Recibido: 10 de junio, $2020 \bullet$ Aprobado: 20 de agosto, 2020

Cómo citar: Pacheco Coello F, Pinto-Catari I, Ramirez-Azuaje D, Orosco-Vargas C, Peraza-Marrero M. Evaluación in vitro de la actividad antihemolítica de extractos acuosos (Camellia sinensis, Hibiscus sabdariffa Linn), bajo un diseño factorial 2x5. cysa [Internet]. 23 de febrero de 2021 [citado 23 de febrero de 2021];5(1):5-10. Disponible en: https://revistas.intec.edu.do/index.php/cisa/article/ view/2073

\section{Resumen}

Introducción: el uso y el estudio de extractos obtenidos de material vegetal se ha incrementado a nivel mundial gracias a sus diversas propiedades bilógicas, como antimicrobiana, anticancerígena, antiinflamatoria y una muy poca estudiada, la antihemolítica.

Objetivo: la investigación tuvo como propósito caracterizar y evaluar la actividad antihemolítica de dos extractos acuosos obtenidos de $C$. sinensis e $H$. sabdariffa.

Material y métodos: para la obtención de los extractos se utilizó hojas de té verde orgánico y cálices deshidratados. Posterior a esto se evaluó la actividad antihemolítica con una suspensión de glóbulos rojos colocando alícuotas de cada extracto a distintas concentraciones en presencia de peróxido de hidrógeno $\left(\mathrm{H}_{2} \mathrm{O}_{2}\right)$.

\footnotetext{
a Analista del Laboratorio de Metales Pesados-Solventes Orgánicos, Docente del Departamento de Ciencias Básicas-Escuela de Bioanálisis, Investigador del Laboratorio de Bioquímica Farmacológica "Dr. Francisco J. Triana Alonso" (BIOMED-UC), estado Aragua, Venezuela. ORCID: 0000-0002-2765-4069, Correo-e: fpacheco2@uc.edu.ve

b Investigadora del Laboratorio de Metales Pesados-Solventes Orgánicos, Toxicología, estado Aragua, Venezuela.

ORCID: 0000-0002-9333-9147, Correo-e: ibispintoc@gmail.com
}

\begin{abstract}
Introduction: the use and study of extracts obtained from plant material has increased worldwide thanks to their various bilogical properties, such as antimicrobial, anticancer, anti-inflammatory and a very little studied anti-haemolytic.

Objective: the research was aimed at characterizing and evaluating the anti-hemolytic activity of two aqueous extracts obtained from $C$. sinensis and $H$. sabdariffa.

Material and methods: Organic green tea leaves and dehydrated calyxes were used to obtain the extracts. After this, the anti-haemolytic activity was evaluated with a suspension of red blood cells by placing aliquots of each extract at different concentrations in the presence of hydrogen peroxide $\left(\mathrm{H}_{2} \mathrm{O}_{2}\right)$.

\footnotetext{
c Investigadora del Laboratorio de Metales Pesados-Solventes Orgánicos, Toxicología, estado Aragua, Venezuela.

ORCID: 0000-0002-4947-8027, Correo-e: doralysramirez@gmail.com

d Investigadora del Laboratorio de Metales Pesados-Solventes Orgánicos, Toxicología, estado Aragua, Venezuela. ORCID: 0000-0002-3173-1004 Correo-e: mmm1504peraza@gmail.com

'Investigadora del Laboratorio de Metales Pesados-Solventes Orgánicos, Toxicología, estado Aragua, Venezuela. ORCID: 0000-0001-5248-9854
} Correo-e: cpovo4@gmail.com
\end{abstract}


Resultados: se observó inhibición de la hemólisis en ambos extractos con una mayor actividad antihemolítica en el extracto de C. sinensis $(\mathrm{p}<0,05)$.

Conclusiones: la concentración de compuestos bioactivos presentes en los extractos favorece y contribuye con los sistemas propios de la célula encargados de eliminar moléculas que afectan el balance redox.

Palabra clave: hemólisis; compuestos fenólicos; Camellia sinensis; Hibiscus sabdariffa.

\section{Introducción}

Las plantas con interés medicinal se han utilizado desde tiempos antiguos para el tratamiento de una diversidad de afecciones, por lo que se consideran en tiempos actuales como una alternativa real ${ }^{1}$. El cuerpo humano tiene un complejo sistema enzimático y no enzimático de defensas antioxidantes que evitan los efectos nocivos, pero muchas veces resulta insuficiente para neutralizar los radicales libres, por ello es necesario acudir a la dieta con antioxidantes de origen natural, como es el caso de algunas especies medicinales cuyos fitoconstituyentes son fuentes de antioxidantes ${ }^{2,3}$. Las potencialidades y beneficios a la salud de Camellia sinensis e Hibiscus sadbariffa, son atribuidas a los compuestos bioactivos que estas poseen (catequinas y flavonoides, antocianinas y otros) capaces de actuar en los procesos redox a nivel celular. En tal sentido, diversos estudios han demostrado que los extractos acuosos e hodroahcohólicos de $C$. sinensis e $H$. sadbariffa, poseen actividad biológica, como antimicrobiana, antiinflamatoria, anticancerígena $\mathrm{y}$ antioxidante, principalmente $e^{4,5,6}$.

Los eritrocitos son un modelo celular utilizado en investigación de daño oxidativo a nivel de las
Results: inhibition of hemolysis was observed in both extracts with a higher anti-hemolytic activity in the C. sinensis extract $(\mathrm{p}<0.05)$.

Conclusions: the concentration of bioactive compounds present in the extracts favors and contributes to the cell's own systems in charge of eliminating molecules that affect the redox balance.

Keywords: Hemolysis; phenolic compounds; Camellia sinensis; Hibiscus sabdariffa.

biomembranas, puesto que al ser sometidos a un estrés oxidativo, el hierro presente en la hemoglobina es liberado, pudiéndose unir a metabolitos intracelulares y componentes de la membrana ${ }^{7}$. Estos compuestos de hierro pueden reaccionar con oxígeno y generar radicales hidroxilos $(\mathrm{OH} \cdot)$ originando una peroxidación lipídica ${ }^{8}$.

Considerando el alto nivel de consumo de $C$ sinensis e $H$. sabdariffa y teniendo en cuenta que el efecto citotóxico es un factor importante para el uso de los extractos naturales, se plateó como objetivo evaluar la capacidad antihemolítica de extractos obtenidos de hojas de té verde orgánico (C. sinensis) y cálices deshidratados de flor o rosa de jamaica ( $H$. sabdariffa), representando este el primer estudio en Venezuela y en Latinoamérica que evalúa estas dos especies simultáneamente.

\section{Material y métodos}

\section{Material vegetal}

El material vegetal empleado en el estudio correspondió a hojas de té verde orgánico (procedencia China) y cálices orgánicos deshidratados de un cultivo propio de los investigadores (figuras 1A y 1B). 

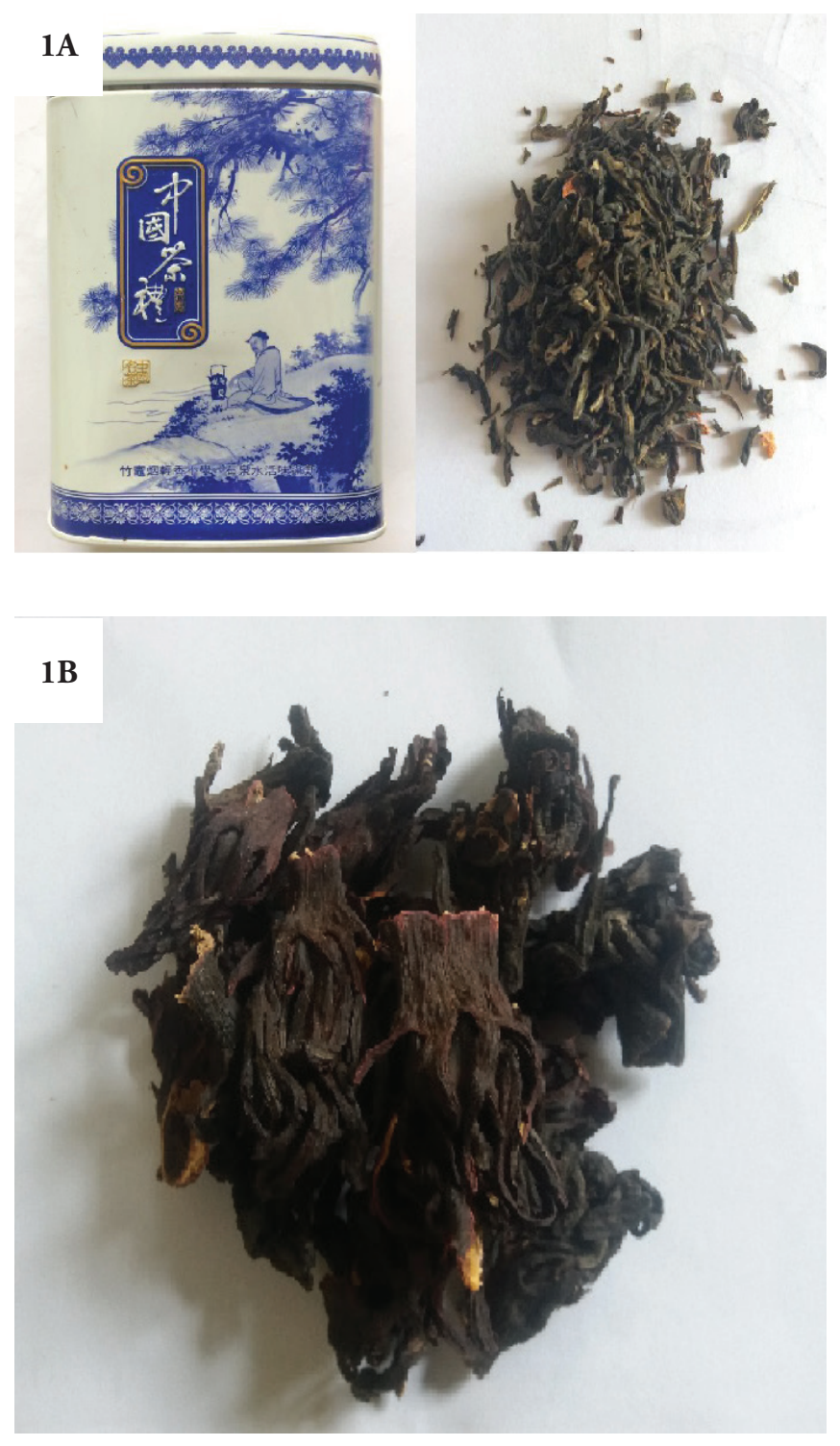

Figura 1. A) Empaque original y hojas de té verde orgánico, B) cálices orgánicos deshidratados

Fuente: elaboración propia.

\section{Obtención de los extractos acuosos}

Los extractos se prepararon con 2,5 g de material vegetal y $100 \mathrm{~mL}$ de agua destilada. Se dejó hervir por $15 \mathrm{~min}$, se separó el líquido del resto de material vegetal por decantación y la extracción se repitió en las mismas condiciones por triplicado. Los extractos se filtraron con papel Whatman No. 1 y se aforó a $200 \mathrm{~mL}$ con agua destilada? .

\section{Extracción sanguínea y preparación de la suspen- sión de eritrocitos}

Siguiendo los lineamientos de la Declaración de Helsinki y del Consejo Técnico del Centro de Estudio en Salud de los Trabajadores del Universidad de Carabobo y previo consentimiento informado, se realizó la extracción sanguínea de sangre periférica de un paciente sin patológica de base. Lo eritrocitos se obtuvieron mediante gradiente de densidades con Ficoll- Hipaque, por centrifugación a $2000 \mathrm{rpm}$, por $3 \mathrm{~min}$, se tomaron con sumo cuidado. Se resuspendieron en PBS glucosado para luego ser centrifugados a $1500 \mathrm{rpm}$, por 12 minutos ${ }^{10}$.

\section{Actividad antihemolítica en presencia de peróxido de hidrógeno $\left(\mathrm{H}_{2} \mathrm{O}_{2}\right)$}

Un volumen de $200 \mu \mathrm{L}$ de suspensión de glóbulos rojo $\left(2,5 \times 10^{5}\right.$ eritrocitos $\left./ \mathrm{mL}\right)$ se incubaron con $1,8 \mathrm{~mL}$ de extracto a las concentraciones señaladas, e incubados a $37^{\circ} \mathrm{C}$, en agitación constante, por $325 \mathrm{~min}$, en presencia de $\mathrm{H}_{2} \mathrm{O}_{2} 5 \%$ (v/v); después de este tratamiento, se centrifugó a 8.000 rpm por $12 \mathrm{~min}$ y el sobrenadante fue usado para medir la hemoglobina liberada. Un blanco de $100 \%$ de hemólisis fue usado $\left(2,5 \times 10^{5}\right.$ eritrocitos tratado con $\mathrm{H}_{2} \mathrm{O}_{2}$ ). El porcentaje de liberación de hemoglobina fue determinado a una longitud de onda $540 \mathrm{~nm}$ empleando el equipo de absorción molecular Génesis 20 (Thermo Scintific) ${ }^{11,12}$.

\section{Diseńo experimental}

Para el proceso de extracción se trabajó con un diseño estadístico factorial $2 \times 5$, considerando dos especies vegetales y cinco concentraciones (50, 100, 150, 200 y $250 \mu \mathrm{g} / \mathrm{mL}$ ) de los extractos caracterizados correspondiente a compuestos fenólicos totales. Cada tratamiento se realizó por triplicado.

\section{Análisis estadístico}

Se aplicó un análisis de varianza de dos vías con interacción (ANOVA), usando el programa Statistix 9.0 para Windows. 


\section{Resultados}

La figura 2 muestra el porcentaje de inhibición de los dos extractos evaluados en un rango de concentración de 25 a $250 \mu \mathrm{g} / \mathrm{mL}$. Se observa que el extracto de C. sinensis presentó un menor \% de hemolisis de los glóbulos rojos en presencia de $\mathrm{H}_{2} \mathrm{O}_{2}$. Al comparar el porcentaje de inhibición el análisis de varianza arrojó significancia estadística en 200 y $250 \mu \mathrm{g} / \mathrm{mL}$ $(\mathrm{p}=0,432$ y $\mathrm{p}=0,465)$.

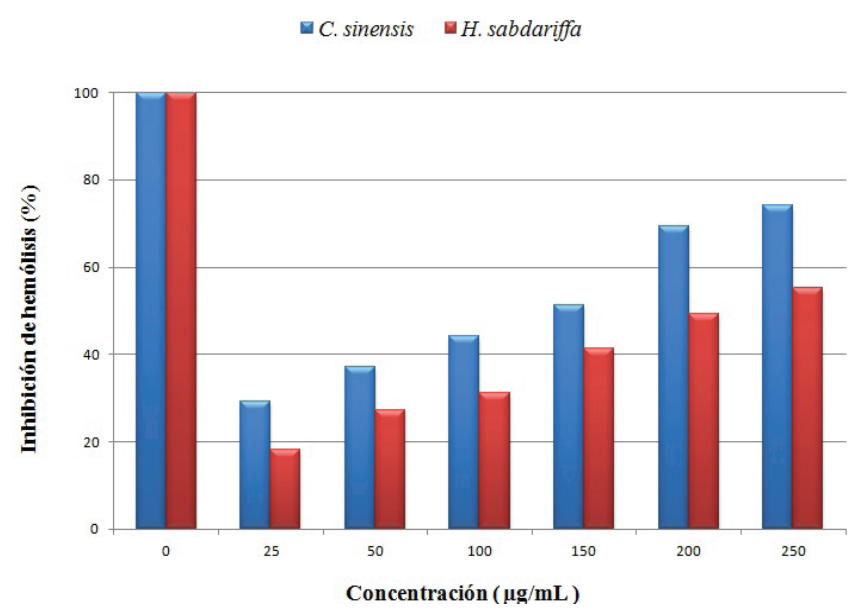

Figura 2. Inhibición de la hemólisis de los extractos acuosos de C. sinenesis e H. Sabdariffa. *Significativo $(p \leq 0,05)$

Fuente: elaboración propia.

\section{Discusión}

Con el propósito de evaluar la actividad antihemolítica de los extractos ya mencionados, se expuso una suspensión de glóbulos rojos a una concentración de variable de cada extracto en presencia de $\mathrm{H}_{2} \mathrm{O}_{2}$ como agente oxidante. En este sentido, ambos extractos presentaron actividad antihemolítica, lo cual puede estar asociado a lo descrito en diversos estudios que señalan que los compuestos fenólicos, en especial flavonoides y catequinas, juegan un papel fundamental en la eliminación de especies reactivas de oxígenos (ROS) asociados a su estructura molecular, actuando sobre enzimas como la catalasa (CAT), glutatión reductasa (GR) y la súper oxido dismutasa
(SOD), manteniendo la integridad de la membrana celular (figura 3) $)^{13,14,15}$.

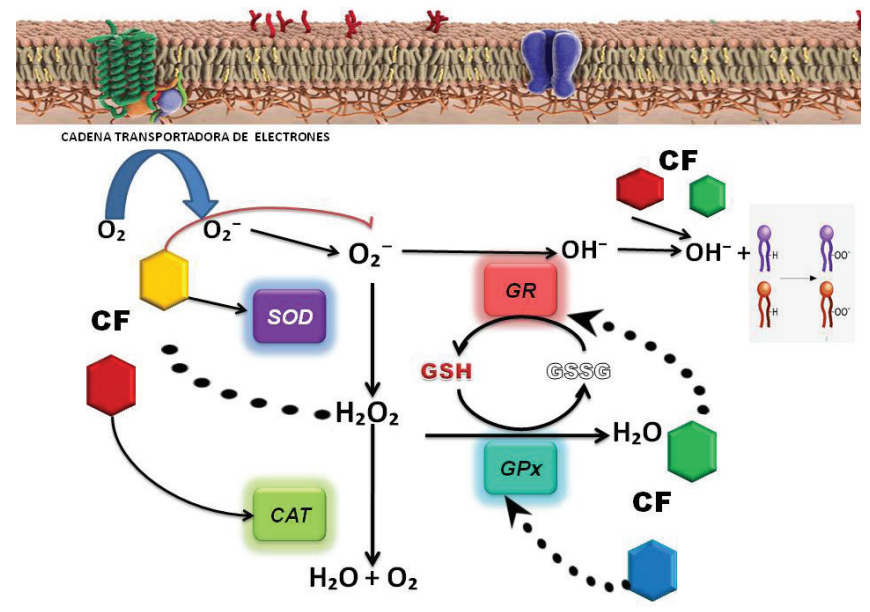

Figura 3. Efecto de los compuestos fenólicos (CF) en el sistema enzimático celular. Los hexágonos en colores representan los CF.

La diferencia apreciable de la actividad evaluada del extracto de $C$. sinencis respecto al de $H$. sabdariffa se debe, fundamentalmente, al mayor contenido de compuestos con actividad antioxidante como la epicatequina, galato de epicatequina, epigalocatequina y galato de epigalocatequina ${ }^{16}$.

Por otra parte, estos resultados son comparables con el hallado en extractos metanólicos de corteza de mango y guayaba, los cuales no mostraron citotoxicidad e inhibieron la hemólisis por encima del 60 $\%$ en presencia de $\mathrm{H}_{2} \mathrm{O}_{2}{ }^{17}$. Asimismo, los extractos hidroalcohólicos de café verde y tostado también han mostrado actividad antihemolítica, con la salvedad de que al ser un material vegetal que debe ser sometido a tostado, la actividad disminuye por efecto de la temperatura ${ }^{18}$. Nabavi et al. $(2010)^{19}$ evaluaron la actividad antihemolítica y antioxidante de extractos hidroalcohólicos de flores, tallos y hojas de Ferula gummosa Boiss hallando una mejor actividad captadora de radicales libres quelatante y actividad antihemolítica dependiendo de la parte de la planta utilizada. Los autores concluyen entre los extractos, las flores contienen el mayor contenido de fenoles y flavonoides. 


\section{Conclusiones}

La diferencia del material vegetal evaluado es un condicionante del efecto observado en este estudio. Emplear el mismo rango de compuestos fenólicos totales en ambos extractos y basados en estudios de caracterización de estas dos especies, nos permite inferir que la actividad antihemolítica evaluada, depende la presencia de moléculas capaces de reducir el estrés oxidativo.

\section{Referencias}

1. Hernández A, Hermosilla V. (2014). Efecto de la concentración de saponinas en la actividad hemolitica de extractos de ocho plantas de uso medicinal en Guatemala. (Tesis de maestría). Universidad de San Carlos de Guatemala, Guatemala. Recuperado de http://www.repositorio.usac.edu.gt. $324 \mathrm{pdf}$

2. Alam M, Bristi N, Rafiquzzaman M. Review on in vivo and in vitro methods evaluation of antioxidant activity. Rev. Saudi. Pharm. Jour. 2013;21(2):143-52.

3. Jiménez $A, N$ Núnez $S$. Terapia antioxidante, estrés oxidativo y productos antioxidantes: retos y oportunidades. Rev. Cub. Sal. Públ. 2011;37 (1):644-60.

4. Hashimoto T, Goto M, Sakakibara H, Oi N, Okamoto M, Kanazawa K. Yellow tea is more potent than other types of tea in suppressing liver toxicity induced by carbon tetrachloride in rats. Phytother Res, 2007;21:668-70

5. Ghazala R, Rajni C. A review on phytochemistry and therapeutic uses of Hibiscus sabdariffa L. Biomedicine Pharmacothe. 2018;102(1):575-6.

6. Quideau S, Deffieux D, Douat-Casassus C, Pouységu L. Plant polyphenols: chemical properties biological activities, and synthesis. Angew Chem Int. 2011;50:586-621.
7. Suwalsky M, Vargas P, Avello M, Villena F, Sotomayor C.P. Human erythrocytes are affected in vitro by flavonoids of Aristotelia chilensis (Maqui) leaves. Int. J. Pharmaceut. 2008;363 (1-2):85-90.

8. Ugartondo V, Mitjans M, Lozano C, Torres JL, Vinardell MP. Comparative study of the cytotoxicity induced by antioxidant epicatechin onjugates obtained from grape. J. Agr. Food Chem. 2006;54(18):6945-50.

9. Reyes-Luengas A, Salinas-Moreno Y, Ovando-Cruz M, Arteaga-Garibay R. Análisis de ácidos fenólicos y actividad antioxidante de extractos acuosos de variedades de jamaica (Hibiscus Sabdariffa L.) con cálices de colores diversos. Agrociencia. 2015;49:277-90

10. Pinell G, Terán C, Guarachi E, Illanes J. Actividad citotóxica in vitro en líneas celulare y células de sangre periférica humana de los alcaloides totales de corteza de Galipea longiflora (Evanta). Rev. Bol. Quim, 2009;26(2):96-100.

11. Goyal M, Singh P, Alam A, Das SK, Igbal MS, Dey S, Bindu S, Pal C, Das SK. Aryl aryl methyl thio arenes prevent multidrug-resistant malaria in mouse by promoting oxidative stress in parasite. Free Radical Biol. Med, 2012;53(1):129-42.

12. Grinberg L, Newmark H, Kitrossky N, Rahamim E, Chevion M, Rachmilewitz E. Protective effects of tea polyphenols against oxidative damage to red blood cells. Biochem. Pharmacol. 1997;54(9):973-878.

13. Herranz-López M, Olivares-Vicente M, Encinar J, Barrajón-Catalán E, Segura-Carretero A, Multi-Targeted Molecular Effects of Hibiscus sabdariffa Polyphenols: An Opportunity for a Global Approach to Obesity. Nutrients. 2017; 907(9):1-26 
14. Peluso I, Serafini M. Antioxidants from black and green tea: from dietary modulation of oxidative stress to pharmacological mechanisms. $\mathrm{Br} \mathrm{J}$ Pharmacol. 2017;174: 1195-208.

15. Zhao CN, Tang GY, Cao S.Y. Phenolic Profiles and Antioxidant Activities of 30 Tea Infusions from Green, Black, Oolong, White, Yellow and Dark Teas. Antioxidants. 2019;8(7): 200-15.

16. Serafini M, Del Rio D, Yao DN, Bettuzzi S, Peluso I. Chapter. Health benefits of tea. In: Benzie IFF, Wachtel-Galor S (2eds). Herbal Medicine: Biomolecular and Clinical Aspects, CRC Press/Taylor \& Francis. USA: Boca Raton; 2011.p. 12-13

17. Durán M, Montero P, Marrugo Y. Extractos metanólicos de corteza de guayaba (Psidium guajava L.) y mango (Mangifera indica L.): efecto citotóxico, antihemolítico y en la morfología de membrana de eritrocitos. Rev. U.D.C.A Act. \& Div. Cient. 2013;16(2):327-34.
18. Abanto S, Tocas M. (2018). Efecto antioxidante de los extractos hidroalcohólicos de los granos verdes y tostados de Coffea arabica L. "café". (Tesis de grado). Universidad privada Antonio Guillermo Urrelo, Perú. Recuperado de http://repositorio. upagu.edu.pe/pdf

19. Nabavi S, Ebrahimzadeh A, Nabavi SM, Eslami B. Antioxidant activity of flower, stem and leaf extracts of Ferula gummosa Boiss. Grasas y Aceites, 2010;61(3):244-50 